\title{
Gel-immobilized single-crystal-like colloidal crystal films
}

\author{
Toshimitsu KANAI ${ }^{\dagger}$ \\ Yokohama National University, 79-5 Tokiwadai, Hodogaya, Yokohama 240-8501, Japan
}

Colloidal crystals, three-dimensional periodic arrays of monodisperse colloidal particles, have attracted considerable attention in the field of materials science and for technological applications because of their novel optical applications as photonic crystals. Although polycrystalline colloidal crystals can be easily prepared, the fabrication of a large single crystal is a challenging for advanced studies pertaining to optical properties or applications. This review reports a unique method for fabricating gelimmobilized single-crystal-like colloidal crystal films and their excellent optical properties. A suspension of charged colloids is forced to flow in a flat capillary cell using an air-pulse-drive system. Optical characterizations such as ordinary spectroscopy, laser Kossel diffraction, and imaging spectroscopy for the crystals indicate that the strong shear flow generated in the cell induces a single-domain crystal with a fixed crystallographic orientation determined by the cell geometry. The flow-aligned crystal can be subsequently immobilized in a self-standing hydrogel film by a photopolymerization technique. The resultant gel-immobilized colloidal crystal film can be used as tunable photonic crystals in nonvolatile ionic liquids and be converted into a dry film of densely packed colloidal crystals without cracks. The present materials are expected to contribute to the development of novel colloidal photonic crystals and open the door to industrial mass production of colloidal photonic crystals.

(2012 The Ceramic Society of Japan. All rights reserved.

Key-words : Colloidal crystals, Photonic crystals, Single crystal, Hydrogel

[Accepted December 26, 2011]

\section{Introduction}

Three-dimensional periodic arrays of monodisperse colloidal particles are called colloidal crystals, by analogy to atomic crystals. ${ }^{1)-10)}$ Colloidal crystals have attracted considerable attention in the field of materials science and for technological applications because of their novel optical applications as photonic crystals. ${ }^{11-13)}$ The spatial periodicity of the refractive index of the colloidal crystalline arrays results in an optical stop band effect, and, hence, they act as photonic crystals in the optical regime. They are potentially useful as non-bleachable color materials, lasers, tunable photonic crystals, etc. ${ }^{14)-17)}$ Although polycrystalline colloidal crystals can be easily prepared, the fabrication of a large single crystal is usually time consuming and difficult. For realizing such attractive applications, a practical method for fabricating the large single crystal is required.

Colloidal crystals may be classified into two categories on the basis of their packing: tightly and loosely packed. The former consists of opal-type colloidal crystals, where the particles are aligned with the closest packing in a dry state. ${ }^{1)-5)}$ The latter consists of charge-stabilized colloidal crystals where the loosely packed particle arrays are suspended in a liquid medium due to the electrostatic repulsive interaction between particles. ${ }^{6)-9)}$ Although the opal-type colloidal crystals have been extensively studied, the fabrication of large single crystals is usually difficult. In particular, crack networks, ranging up to a few hundred microns in size, are generally formed during the drying process, which causes significant deterioration of the optical properties of the materials. On the other hand, in the charge-stabilized system, the possibility of forming large single crystals seems to be high due to strong, long range interactions between particles. Although the colloidal crystalline arrays in the liquid medium

\footnotetext{
Corresponding author: T. Kanai; E-mail: tkanai@ynu.ac.jp
}

don not exhibit a high degree of stability against external disturbances such as mechanical vibrations and chemical contamination, approaches to immobilize the ordered arrays in a hydrogel by the polymerization of gelation reagents in the liquid medium have been examined. Furthermore, if the loosely packed particle arrays are immobilized in the gel, highly tunable photonic crystals, which are very attractive for practical applications, can be obtained. ${ }^{18)-24)}$

In this review, a unique method for fabricating gel-immobilized single-crystal-like colloidal crystal films by the combination of a shear-induced method and a photopolymerization technique is reported. A suspension of charged colloids is forced to flow in a flat capillary cell. The strong shear flow generated in the cell induces long-range orientational ordering of the crystals. ${ }^{25), 26)}$ The formed particle array can be immobilized in a self-standing hydrogel film using the photopolymerization technique. The obtained gel-immobilized colloidal crystal film can be used as a tunable photonic crystal. In addition, the film can be converted into a dry film of densely packed colloidal crystals without cracks.

\section{Fabrication of a gel-immobilized single-crystal-like colloidal crystal film}

\subsection{Single crystallization by shear flow}

We developed an air-pulse-drive system to form single-crystallike colloidal crystals in a flat capillary cell (Fig. 1). ${ }^{25}$ The capillary cell (thickness: $0.1 \mathrm{~mm}$; width: $9 \mathrm{~mm}$; length: $70 \mathrm{~mm}$ ) is made of fused quartz, and one side of the cell is connected to a digital pulse regulator (Musashi Engineering Inc., ML-606GX) with an air compressor (500 $\mathrm{kPa}$ maximum pressure). A short air pulse generated by electronically controlled valve actions drives the flow of colloidal crystals in the cell. Adjusting the air-pulse pressure ( $\Delta P$, difference from atmospheric pressure) can quantitatively control the strength of the pulsed flow of the colloidal crystals. Various samples were prepared at different $\Delta P$ values, 


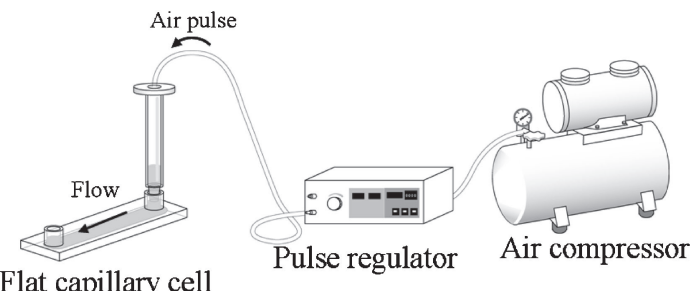

Flat capillary cell

Fig. 1. Schematic diagram of the air-pulse-drive system for fabrication of single-crystal-like colloidal crystals.

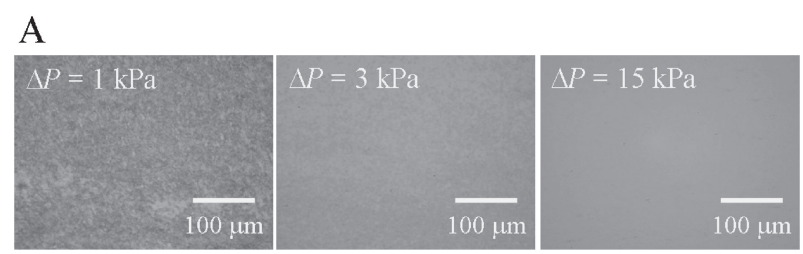

B
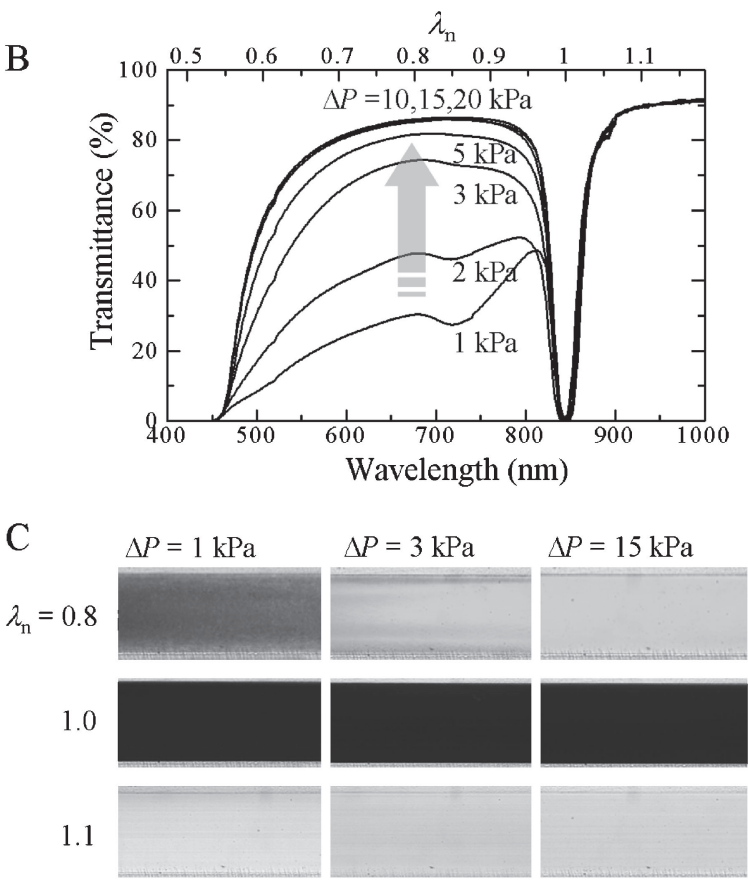

Fig. 2. (A) Transmission optical microscopy images of the polystyrene colloidal crystals prepared at various $\Delta P$ values. The particle diameter is about $200 \mathrm{~nm}$ and the particle concentration is about $10 \%$. (B) Transmission spectra of the colloidal crystals prepared at various $\Delta P$ values. $\lambda_{n}$ represents a value normalized by the dip wavelength, $\lambda_{n}=\lambda / \lambda_{111}$. (C) Single-wavelength images for representative wavelengths for the colloidal crystals prepared at indicated $\Delta P$ values.

and the flow effects on the crystals were investigated as a function of $\Delta P$.

Figure 2(A) shows $\Delta P$-dependent changes in the texture of the polystyrene colloidal crystals observed through an optical microscope under transmission illumination. At low $\Delta P$, the polycrystalline texture is observed. However, upon increasing $\Delta P$, the uniformity in the texture increases, and at $\Delta P=15 \mathrm{kPa}$, the prepared texture appears almost uniform. Figure 2(B) shows the transmission spectra of the colloidal crystals prepared at various $\Delta P$ values when the incident light is normal to the cell face. All samples show a deep dip at $845 \mathrm{~nm}$, which corresponds to the optical stop band caused by the Bragg diffraction from the
A
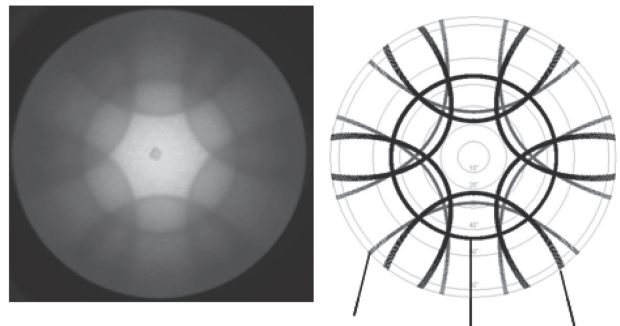

$\mathrm{B}$
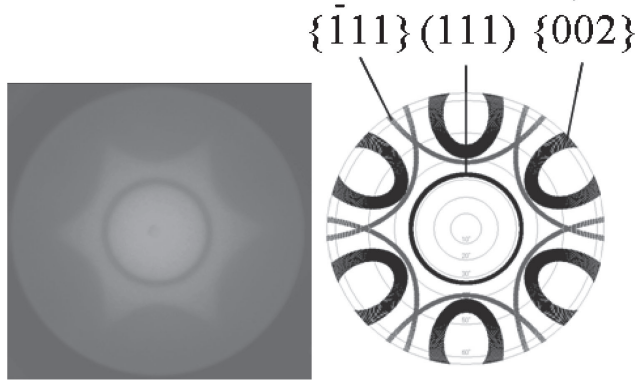

Flow

Fig. 3. Kossel pattern of the colloidal crystals at wavelength of (A) $543.5 \mathrm{~nm}$ and (B) $632.8 \mathrm{~nm}$.

(111) lattice planes of the face-centered-cubic (fcc) structure parallel to the cell surface. Upon increasing $\Delta P$, the transmittance in the region with wavelengths shorter than the dip markedly increases and then saturates at high values at $\Delta P>10 \mathrm{kPa}$. Figure 2(C) shows the spatial distribution of transmittance with brightness at three representative wavelengths - below, at, and above the $\operatorname{dip}\left(\lambda_{n}=0.8,1.0\right.$, and 1.1)-for the same sample, where the incident light is normal to the cell face. In the image, the absence of uniformity in the optical properties is detected from the contrast in brightness. ${ }^{27)}$ For wavelengths above and at the dip, the transmittance of the entire area is very uniform regardless of $\Delta P$. However, for wavelengths below the dip, a nonuniform texture with low brightness is observed at low $\Delta P$, and both uniformity and brightness increase with $\Delta P$.

The observed $\Delta P$ dependence is simply explained as a consequence of the decrease in the fraction of irregular polycrystalline areas with an increase in the flow rate, according to the shear effect. ${ }^{28)-30)}$ Considering the Bragg conditions of the fcc structure, the longest Bragg wavelength occurs when the incident light is normal to the (111) planes, which corresponds to the dip in Fig. 2(B). If a sample includes polycrystalline parts with random orientations, various Bragg reflections occur at wavelengths shorter than the dip. Therefore, a reduction in transmittance occurs in the shorter-wavelength region but not in the longer-wavelength one. Upon increasing $\Delta P$, the polycrystalline domains of the colloidal crystals are aligned by the shear flow in the cell, resulting in an increase in the transmittance. The saturation behavior above $\Delta P=10 \mathrm{kPa}$ indicates that the polycrystalline domains are converted to the single-crystallike structure.

The crystal shows a distinct laser diffraction pattern, i.e., Kossel pattern (Fig. 3). ${ }^{31)}$ Clear hexagonally symmetric patterns are observed, and the same patterns are observed at different parts of the sample. Quantitative analysis of these data indicates that almost the entire capillary space is filled with a single-domain crystal with a fixed crystallographic orientation determined by the 
cell geometry. The crystal has a cubic-close-packed structure of colloids with stacking faults or a twinned fcc structure with a fixed orientation; the fcc (111) lattice plane is parallel to the cell face, and one of the closest packed directions in the (111) plane is parallel to the cell axis.

Since the fabrication method mostly relies on the physical phenomenon of the shear-induced effect, it has wide applicability under other conditions. In fact, we have succeeded in obtaining single-crystal-like colloidal crystals similar to those mentioned above with other particle sizes and particle volume concentrations, and with silica particles. ${ }^{32)}$

\subsection{Immobilizing the single-crystal-like colloidal crystals in a gel film}

The single-crystal-like colloidal crystals prepared by shear flow can be immobilized in a hydrogel film through photopolymerization of gelation reagents $-N$-methylol-acrylamide as a monomer, $N, N^{\prime}$-methylene-bis-acrylamide as a crosslinker, and 2,2'-azobis[2-methyl- $N$-(2-hydroxyethyl)propionamide] as a photoinduced polymerization initiator for ultraviolet (UV) light—added to the starting colloidal suspension. ${ }^{33)}$ Figure 4(A) shows the transmission spectra of the colloidal crystals before and after gelation. By properly adjusting the conditions for gelation, including concentration, UV irradiation intensity, and time, the excellent spectral profile, i.e., the deep dip due to the stop band and high transmittance at the pass-band wavelength, can be preserved after gelation. In addition, single wavelength images indicate that high uniformity over a large area is preserved after gelation [Fig. 4(B)]. The obtained gel film is sufficiently solid to be handled with tweezers and can be removed from the fabrication cell as a self-standing film [Fig. 4(C)]. The film thickness is about $0.1 \mathrm{~mm}$ and includes over 300 particle layers. Since the curved surface of the gel film is illuminated from various angles by using room lamps, it shows multiple iridescent colors.

\section{Tuning the effective width of the optical stop band in gel-immobilized colloidal crystals}

The optical stop band in colloidal crystals is characterized by the optical stop band wavelength and the bandwidth. Although the former is known to be highly tunable by changing the lattice constant, the latter is intrinsically determined by the refractive index contrast between the particles and the surrounding medium. Therefore, controlling the bandwidth, which is important for optical applications, is difficult. However, the effective bandwidth in gel-immobilized colloidal crystals can be tuned by controlling the photopolymerization conditions for immobilizing the colloidal crystals in the gel network. ${ }^{34)}$ Figures 5(A) and 5(B) show temporal changes in the transmission spectra of the colloidal crystals during equilibration after light irradiation for 3 and $1 \mathrm{~h}$, respectively. In the case of the 3-h irradiation, the initial spectrum does not change, at least within the experimental time range, i.e., $50 \mathrm{~h}$. On the other hand, in the case of the 1-h irradiation, the (111) dip gradually expands during equilibration in the dark after the irradiation is complete while the spectrum profile except for the dip region is preserved, i.e., high transmittance at the pass band and the absence of band edge shift at approximately $450 \mathrm{~nm}$ are observed; this gives the impression that the optical stop band is expanded.

The dip expansion observed in this study can be explained if the gel possesses some inhomogeneity. The lattice structure of the colloidal crystals immobilized in the inhomogeneous gel network would become inhomogeneous as a result of subsequent swelling equilibration. This implies that the interplanar spacing of the
(111) lattice planes, which determines the dip wavelength, is not constant but fluctuates around a value for the homogeneous case. If the length scale of such an inhomogeneity is smaller than the spatial resolution of the spectral measurement $(100 \mu \mathrm{m})$ in the lateral direction, the spectrum of the sample would be observed as the superposition of spectra with different dip wavelengths, resulting in an apparent dip expansion. Since incomplete polymerization reaction is the cause of inhomogeneity in gels, an increase in the reaction time, i.e., irradiation time, would reduce the inhomogeneity. Although the dip expansion due to inhomogeneity is essentially different from the expansion of the optical stop band in a perfect crystal, it has an equivalent effect on the apparent spectral property. ${ }^{35), 36)}$

Another interesting feature is that dip expansion can be frozen by additional light irradiation. For example, if the sample is first irradiated for $1 \mathrm{~h}$, retained in the dark for $7 \mathrm{~h}$, and irradiated again for $3 \mathrm{~h}$, the expansion of the width can be frozen at $72 \mathrm{~nm}$ by the second irradiation. In the present material, the dip width can be tuned in the range $46-156 \mathrm{~nm}$.

\section{Dry film of colloidal crystals without cracks}

The obtained gel-immobilized colloidal crystal film, which includes water, can be converted into a dry film of densely packed colloidal crystals while maintaining excellent optical quality (Fig. 6). ${ }^{37)}$ Since particles are bounded by polymer networks in the gelled colloidal crystal film, it seems possible to obtain dry colloidal crystals by removing the water without destroying the particle arrangement structure. However, simply drying the gel film in air destroys the crystalline structure of the particles owing to the inhomogeneous shrinkage of the gel. To suppress the inhomogeneous shrinkage and deformation of the gel, a circular fragment (diameter: $7 \mathrm{~mm}$; thickness: $0.1 \mathrm{~mm}$ ) cut from the wet gel film is soaked in aqueous solutions with increasing concentrations from 0 to $100 \%$ (in increments of $1-2 \mathrm{vol} \%)$ of ethanol (EtOH), which is a solvent with poor affinity for the gel-polymer, and then, it is dried in air. After the processes, the film was finally contracted down to $52 \%$ in linear scale, corresponding to a particle volume fraction of about $73 \%$ that almost achieves the sphere-touching condition. The resultant dried film shows an even, round shape with a remarkable diffraction color [Fig. 6(A)], indicating that isotropic contraction preserving the crystalline order is achieved. The regularity of the particle arrays was observed through scanning electron microscopy [Figs. 6(C) and 6(D)]. The particles covered with gels are ordered well and exhibit a 6-fold symmetry. It should be noted that an optical microscope and the scanning electron microscope show that the dry colloidal crystal film has no cracks at any scale. Figure 7(A) shows the transmission spectra of the gel film at the initial stage (swelled in water), after replacement with $\mathrm{EtOH}$, and after drying, where the incident light is normal to the film surface. The shift in the dip wavelength is caused by the contraction or reduction in the lattice spacing and the change in the refractive index of the medium. All of the spectra show a sharp dip at the stop band and high transmittance at the pass-band wavelength, suggesting that the crystallinity of the gel film is preserved throughout the process not only at the surface but also across its thickness. The advantage of this material is that the Bragg wavelength or optical stop-band wavelength of the colloidal crystals can be easily tuned by varying the amount of gelation reagent added in the starting colloidal suspension. In conventional synthetic opals where the particles touch each other, the lattice spacing of the crystal is uniquely determined as a function of particle diameter. However, 

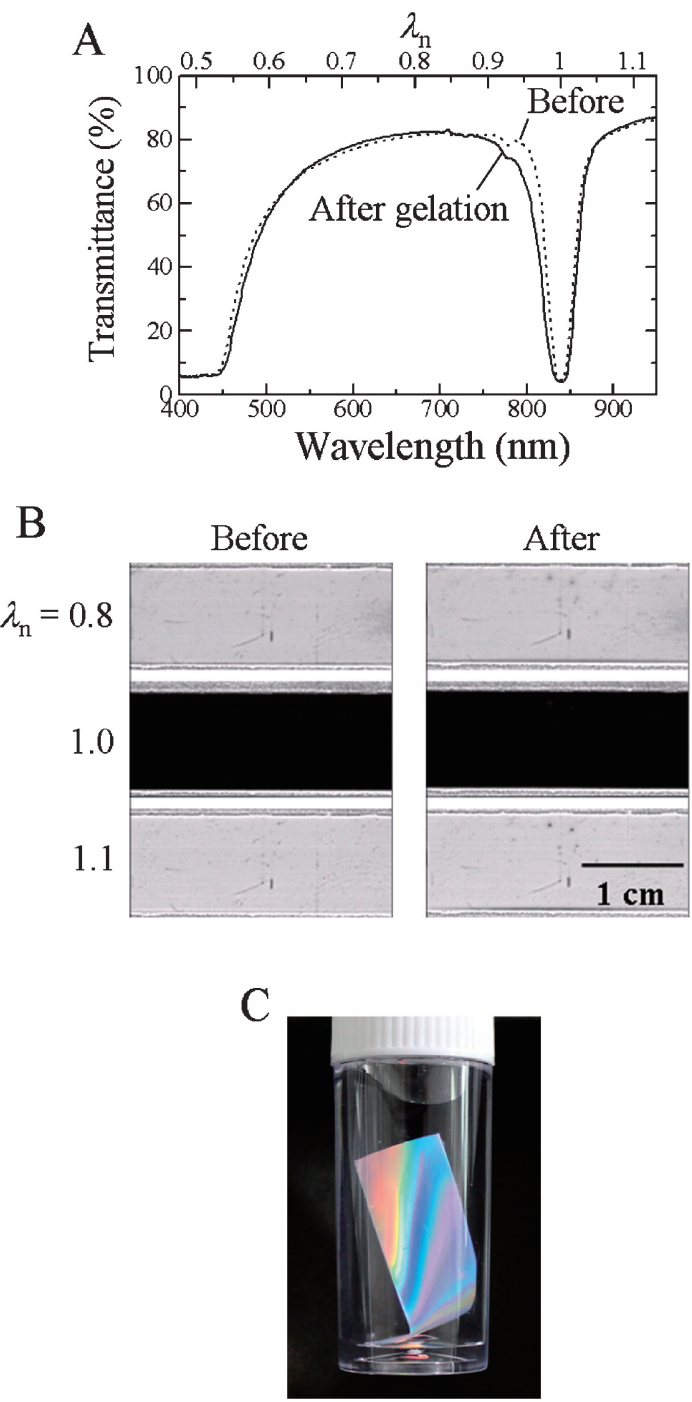

Fig. 4. (A) Transmission spectra of the colloidal crystals before and after gelation. (B) Single-wavelength images taken under the transmission illumination before and after gelation. The incident light is normal to the cell face. (C) Photograph of the gel-immobilized colloidal crystal film soaked in water.

for the present dry colloidal crystals, the lattice spacing can be adjusted simply by varying the amount of gelation reagent. Thus, the Bragg wavelength or optical stop-band wavelength of the colloidal crystals can be tuned over a wide range [Figs. 7(B) and $7(\mathrm{C})]$. This tunability of the stop-band wavelength without changing the particle size is a great advantage, especially for industrial applications.

\section{Tunable colloidal crystals in ionic liquids}

Since hydrogels undergo a volume change in response to external stimuli such as changes in solvent, temperature, and $\mathrm{pH}$, the lattice constant or optical stop band of the colloidal crystals embedded in these gels can be altered on demand by applying external stimuli; this is potentially useful for applications such as tunable photonic crystals and biological and chemical sensors. ${ }^{18-24)}$ However, the evaporation of the solvent in the gel destabilizes the crystalline structure of the colloids, which is an issue for some applications. We found that ionic liquids, which are nonvolatile at room temperature, can be used
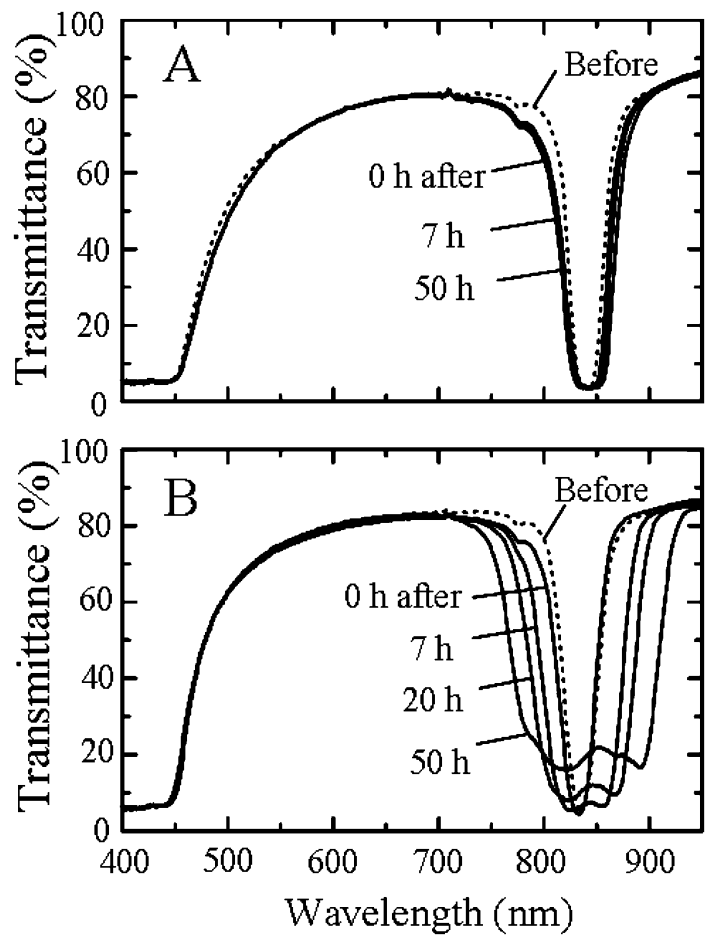

Fig. 5. Temporal changes in transmission spectra of colloidal crystals after light irradiation. Irradiation time is (A) $3 \mathrm{~h}$ and (B) $1 \mathrm{~h}$. The dashed line indicates the spectrum before irradiation.
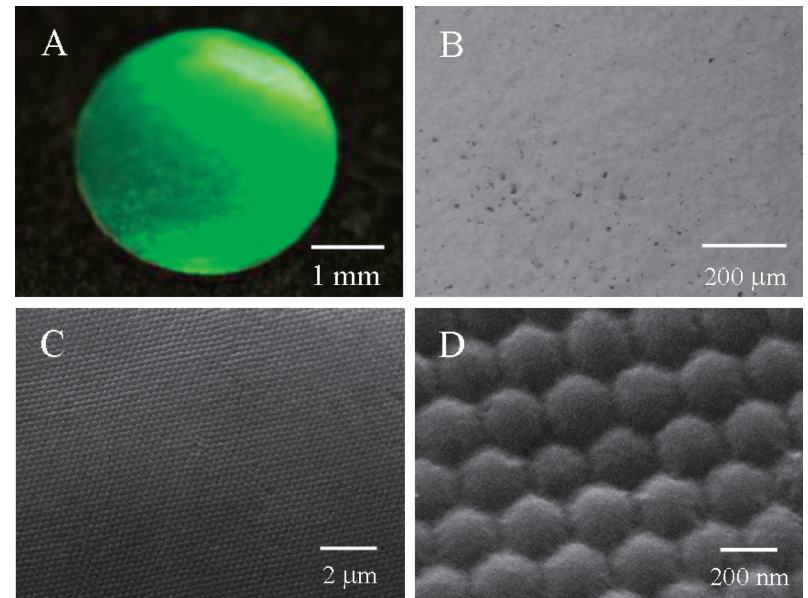

Fig. 6. (A) Photograph of the dried colloidal crystal gel film. Images of the dried colloidal crystal gel film at various scale sizes, observed by an optical microscope (B) and a scanning electron microscope (C-D).

as suitable swelling solvents for gel-immobilized colloidal crystals. ${ }^{38)}$ When the gel film is soaked in hydrophobic ionic liquids such as 1,3-diallylimidazolium bis(trifluoromethanesulfonyl)imide, the gel shrinks considerably, as is the case with most organic solvents. As a result, the crystallinity of the colloids immobilized in the gel is destroyed. On the other hand, when the gel film is soaked in hydrophilic ionic liquids such as 1,3-diallylimidazolium bromide, the crystalline structure of the colloids remains intact. Furthermore, the swelling volume of the gel can be easily tuned by varying the mixing ratio of hydrophilic and hydrophobic ionic liquids. Thus, the Bragg wavelength or optical stop-band wavelength of the colloidal crystals can be adjusted over a wide range. Figure 8(A) shows the reflection 

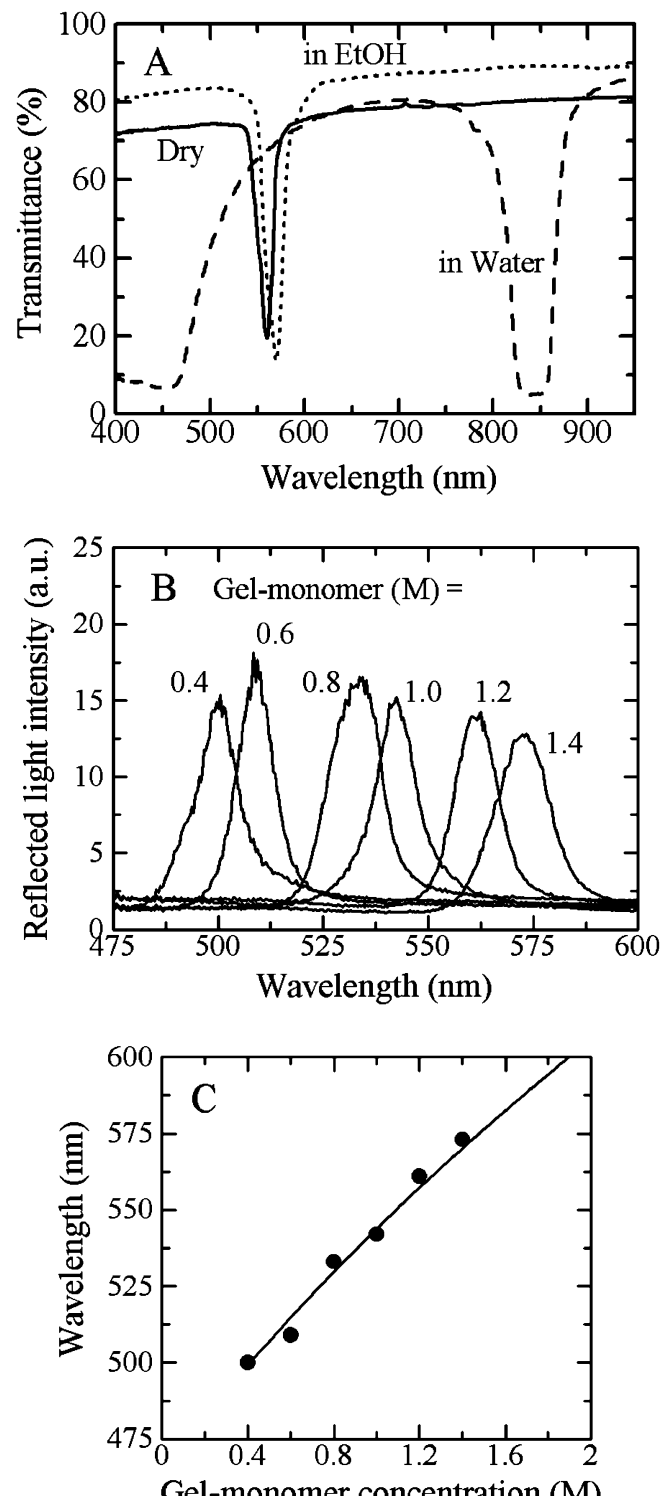

Fig. 7. (A) Transmission spectra of the colloidal crystal gel film swelled in water, shrunk in EtOH, and dried. (B) Normal reflection spectra of the dried colloidal crystal gel films prepared with different gel-monomer concentrations. (C) Plots of the wavelength peaks read from $\mathrm{B}$ as a function of the gel-monomer concentration. The solid line is calculated using the Bragg equation, assuming that the space among particles is filled with gel-polymer.

spectra of the gel-immobilized colloidal crystal film immersed in the mixed solution of the hydrophilic ionic liquid, 1,3-diallylimidazolium bromide, and the hydrophobic ionic liquid, 1,3diallylimidazolium bis(trifluoromethanesulfonyl)imide, for various mixing ratios $x$. Upon decreasing the volume fraction of the hydrophilic ionic liquid, the Bragg reflection peak shifts to a shorter wavelength in a wide range of about $400 \mathrm{~nm}$, preserving the optical quality. In general, the swelling-shrinking phenomenon that depends on the change in the mixing ratio of solvents is known to be a type of phase transition, and the gel size (or Bragg wavelength) is known to be a strongly non-linear function of the mixing ratio, as already reported for the water-ethanol system. ${ }^{33), 39)-41)}$ Surprisingly, however, the Bragg wavelength and swelling size of the present material show linear dependence on the mixing ratio $x$ [Fig. 8(B)]. Although theoretical understand-
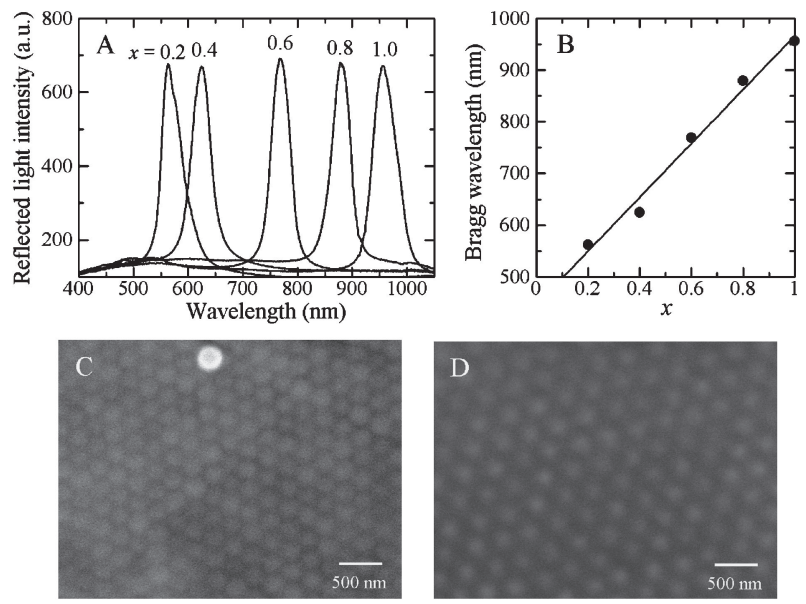

Fig. 8. (A) Reflection spectra of the gel-immobilized silica colloidal crystal film immersed in the mixed solution of 1,3-diallylimidazolium bromide and 1,3-diallylimidazolium bis(trifluoromethanesulfonyl)imide at various mixing ratios. The particle diameter is about $200 \mathrm{~nm}$. The parameter $x$ is the volume fraction of 1,3-diallylimidazolium bromide measured prior to mixing. (B) Bragg wavelength obtained from $\mathrm{A}$ as a function of $x$. Top-view images of gel-immobilized silica colloidal crystal films with $x=0.2$ (C) and $x=0.8$ (D) observed by scanning electron microscopy.

ing of the intriguing linear behavior seems to be a challenging area of research for the future, it is quite advantageous for adjusting the optical characteristics of the crystals in practical applications.

Since ionic liquids do not evaporate even in high vacuum, the distance-controlled particle arrays in the swollen gel can be directly observed by scanning electron microscopy [Figs. 8(C) and 8(D)]. While relatively dense arrays of particles are observed for $x=0.2$, loose arrays of particles are seen for $x=0.8$. This confirms that uniform expansion or shrinkage of the periodic arrays can be successfully achieved by changing the mixing ratio of the two different ionic liquids. Thus, the use of ionic liquids as solvents for gel-immobilized colloidal crystals will increase the possibility of designing colloidal photonic crystals in which there is no solvent evaporation.

\section{Summary}

A method for fabricating a gel film containing single-crystallike colloidal crystals was reported. Single crystallization involving the use of the air-pulse-drive system is simple, fast, and applicable to various kinds of functional particles. In addition, as the present method is suitable for automated processes, it opens the door to industrial mass production of colloidal photonic crystals and is expected to contribute to the development of novel colloidal photonic crystals.

Acknowledgements Part of this work was carried out at National Institute for Materials Science (NIMS), kindly hosted by Dr. Tsutomu Sawada. Financial support by Japan Society for the Promotion of Science [Grant-in-Aid for Young Scientists (A) 22686063] is gratefully acknowledged.

\section{References}

1) K. P. Velikov, C. G. Christova, R. P. A. Dullens and A. van Blaaderen, Science, 296, 106-109 (2002).

2) G. A. Ozin and S. M. Yang, Adv. Funct. Mater., 11, 95-104 (2001). 
3) F. Garcia-Santamaria, H. T. Miyazaki, A. Urquia, M. Ibisate, M. Belmonte, N. Shinya, F. Meseguer and C. Lopez, $A d v$. Mater, 14, 1144-1147 (2002).

4) H. Miguez, F. Meseguer, C. Lopez, A. Mifsud, J. S. Moya and L. Vazquez, Langmuir, 13, 6009-6011 (1997).

5) P. Ni, P. Dong, B. Cheng, X. Li and D. Zhang, Adv. Mater, 13, 437-441 (2001).

6) P. Pieranski, Contemp. Phys., 24, 25-73 (1983).

7) K. Ito, K. Sumaru and N. Ise, Phys. Rev. B, 46, 3105-3107 (1992).

8) "Ordering and Phase Transitions in Charged Colloids", Ed. by A. K. Arora and B. V. R. Tata, VCH, New York (1996).

9) A. P. Gast and W. B. Russel, Phys. Today, 51, 24-30 (1998).

10) S. Furumi, H. Fudouzi and T. Sawada, Laser \& Photon. Rev., 4, 205-220 (2010).

11) E. Yablonovitch, Phys. Rev. Lett., 58, 2059-2062 (1987).

12) S. John, Phys. Rev. Lett., 58, 2486-2489 (1987).

13) J. D. Joannopoulos, R. D. Meade and J. N. Winn, "Photonic Crystals", Princeton University Press, Princeton NJ (1995).

14) K. Sakoda, "Optical Properties of Photonic Crystals", SpringerVerlag, Berlin (2001).

15) D. A. Weitz and W. B. Russel, Eds. MRS Bull., 29, 82-83 (2004).

16) A. Polman and P. Wiltzius, Eds. MRS Bull., 26, 608-610 (2001).

17) D. G. Grier, Ed. MRS Bull., 23, 21-21 (1998).

18) E. A. Kamenetzky, L. G. Magliocco and H. P. Panzer, Science, 263, 207-210 (1994).

19) J. Holtz and S. A. Asher, Nature, 389, 829-832 (1997).

20) H. Fudouzi and Y. Xia, Adv. Mater., 15, 892-896 (2003).

21) S. H. Foulger, P. Jiang, A. Lattam, D. W. Smith, J. Ballato, D. E. Dausch, S. Grego and B. R. Stoner, Adv. Mater., 15, 685689 (2003)

22) Y. Iwayama, J. Yamanaka, Y. Takiguchi, M. Takasaka, K. Ito, T. Shinohara, T. Sawada and M. Yonese, Langmuir, 19, 977980 (2003).
23) T. Kanai, D. Lee, H. C. Shum and D. A. Weitz, Small, 6, 807$810(2010)$

24) T. Kanai, D. Lee, H. C. Shum, R. K. Shah and D. A. Weitz, Adv. Mater., 22, 4998-5002 (2010).

25) T. Kanai, T. Sawada, A. Toyotama and K. Kitamura, $A d v$. Funct. Mater., 15, 25-29 (2005).

26) T. Sawada, Y. Suzuki, A. Toyotama and N. Iyi, Jpn. J. Appl. Phys., 40, L1226-L1228 (2001).

27) T. Kanai, T. Sawada and K. Kitamura, Chem. Lett., 34, 904905 (2005).

28) B. J. Ackerson, Nature, 281, 57-60 (1979).

29) B. J. Ackerson, J. B. Hayter, N. A. Clark and L. Cotter, J. Chem. Phys., 84, 2344-2349 (1986).

30) L. B. Chen, M. K. Chow, B. J. Ackerson and C. F. Zukoski, Langmuir, 10, 2817-2829 (1994).

31) T. Kanai, T. Sawada, I. Maki and K. Kitamura, Jpn. J. Appl. Phys., 42, L655-L657 (2003).

32) T. Kanai, T. Sawada and J. Yamanaka, J. Ceram. Soc. Japan, 118, 370-373 (2010)

33) A. Toyotama, T. Kanai, T. Sawada, J. Yamanaka, K. Ito and K. Kitamura, Langmuir, 21, 10268-10270 (2005).

34) T. Kanai, T. Sawada, A. Toyotama, J. Yamanaka and K. Kitamura, Langmuir, 23, 3503-3505 (2007).

35) P. Jiang, G. N. Ostojic, R. Narat, D. M. Mittleman and V. L. Colvin, Adv. Mater., 13, 389-393 (2001).

36) J. H. Park, W. S. Choi, H. Y. Koo, J. C. Hong and D. Y. Kim, Langmuir, 22, 94-100 (2006).

37) T. Kanai and T. Sawada, Langmuir, 25, 13315-13317 (2009).

38) T. Kanai, S. Yamamoto and T. Sawada, Macromolecules, 44, 5865-5867 (2011).

39) P. J. Flory, "Principles of Polymer Chemistry", Cornell University, Ithaca (1953).

40) T. Tanaka, D. Fillmore, S.-T. Sun, I. Nishino, G. Swislow and A. Shah, Phys. Rev. Lett., 45, 1636-1639 (1980).

41) S. Katayama, Y. Hirokawa and T. Tanaka, Macromolecules, 17, 2641-2645 (1984).

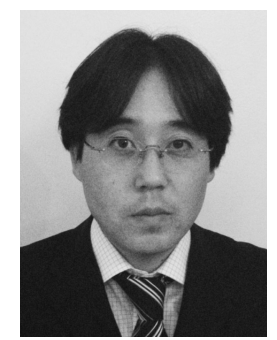

Toshimitsu Kanai received Ph.D. in Engineering from the University of Tokyo in 2002. He worked as a postdoctoral fellow at National Institute for Materials Science from 2002 and at RIKEN from 2006. He joined the research group of Professor David A. Weitz in Harvard University as a postdoctoral fellow from 2007. He has been working as an assistant professor at Yokohama National University from 2009 to present. 